The Israeli Journal of Aquaculture - Bamidgeh, IJ A_69.2017.1392, 13 pages

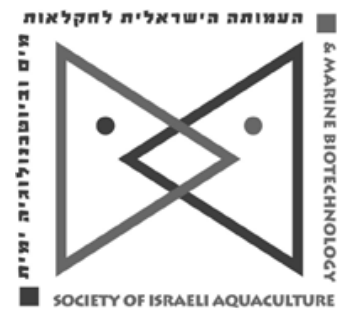

The IJ A appears exclusively as a peer-reviewed on-line open-access journal at http://www. siamb. org.il. To read papers free of charge, please register online at registration form.

Sale of IJ A papers is strictly forbidden.

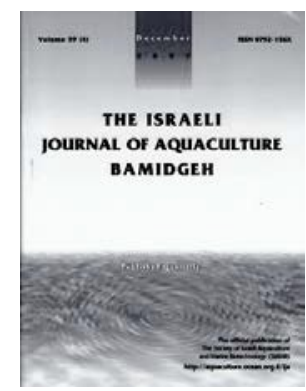

\title{
Molecular cloning of I ndian hedgehog gene and its expression in golden pompano Trachinotus ovatus (Linnaeus 1758) larvae at different water temperatures
}

\author{
Zhenhua Ma ${ }^{1,2,3,4}$, Mingjun Fu' ${ }^{1,2}$, J ing Hu, Rui Yang', Chengyu Wei, \\ Jian G. Qin ${ }^{*}$, Gang Yu'**
}

${ }_{1}^{1}$ Tropical Aquaculture Research and Development Center, South China Sea Fisheries Research Institute, Chinese Academy of Fishery Sciences, Sanya, 572018, P.R. China

${ }^{2}$ Key Laboratory of South China Sea Fishery Resources Exploitation and Utilization, Ministry of Agriculture, Guangzhou, 510300, P.R. China ${ }^{3}$ School of Biological Sciences, Flinders University, GPO Box 2100, Adelaide, SA 5001, Australia

${ }^{4}$ Guangxi Hiseaton Foods Co., Ltd., Fangchenggang 538021, China Key words: Indian Hedgehog gene (I hh); expression; ontogeny; temperature; golden pompano Trachinotus ovatus.

\begin{abstract}
The Indian hedgehog (Ihh) gene in golden pompano larvae was cloned and analyzed. The expression of Ihh during larval fish ontogeny in the first 18 days was examined, and then the expression of Ihh in fish tissues was evaluated on 18 days post hatching (DPH). Subsequently, the response of Ihh to water temperatures of 23, 26, and $29^{\circ} \mathrm{C}$ was compared on $12 \mathrm{DPH}$ and $18 \mathrm{DPH}$. The cDNA sequence length of the golden pompano Ihh gene is $1,484 \mathrm{bp}$ with an open reading frame of $1,314 \mathrm{bp}$. The Ihh gene encodes 437 amino acids and has a calculated molecular weight of $48.175 \mathrm{kDa}$ and a theoretical isoelectric point of 6.22. After hatching, the expression of Ihh increased with fish age and peaked at $3 \mathrm{DPH}$. After fluctuations between 4 and $12 \mathrm{DPH}$, the expression of Ihh reached the second peak at $18 \mathrm{DPH}$. The highest expression of Ihh in tissues occurred in the fish intestine, followed in the stomach on $18 \mathrm{DPH}$. Water temperature significantly affected Ihh expression. On $12 \mathrm{DPH}$, the expression of Ihh at $23^{\circ} \mathrm{C}$ was significantly higher than that at 26 and $29{ }^{\circ} \mathrm{C}$. In contrast, the expression of Ihh at 26 and $29^{\circ} \mathrm{C}$ was significantly higher than that at $23{ }^{\circ} \mathrm{C}$ on $18 \mathrm{DPH}$. This study detected the gene expression of $\mathrm{Ihh}$ at the early stage of golden pompano development and the time-dependent expression of Ihh in fish larvae is important to understand the ontogeny of bone formation in fish larvae.
\end{abstract}

\section{Introduction}

The Hedgehog ( $\mathrm{Hh}$ ) gene family codes for a class of secreted proteins that act in a signaling pathway to transmit information for cell differentiation in embryogenesis and development in both vertebrates and invertebrates (Bijlsma et al., 2004; Ingham and McMahon, 2001). Proteins in the Hh family of vertebrates control cell growth, survival

* Corresponding author. e-mail: jian.qin@flinders.edu.au; gyu0928@163.com 
and development in almost all body tissues (Varjosalo and Taipale, 2008). Hh proteins are composed of two distinct domains: an N-terminal "Hedge" domain and a C-terminal "Hog" domain (Burglin, 2008), and separated during an auto-cleavage reaction to generate two similar-sized globular fragments (Burglin, 2008; Lee et al., 1994). Such special structure enables $\mathrm{Hh}$ proteins to undergo auto-cleavage, and lipid modifications in the endoplasmic reticulum and produce a mature signaling peptide (Beachy et al., 1997; Chen et al., 2004; Chen et al., 2011; Lee et al., 1994), which can be incorporated into lipoprotein particles or diffuse freely to target cells (Eugster et al., 2007; Ingham and McMahon, 2001). In vertebrates, the $\mathrm{Hh}$ genes are classified into three subgroups: Desert Hedgehog (Dhh), Indian Hedgehog (I hh), and Sonic Hedgehog (Shh) (Echelard et al., 1993; Wada and Makabe, 2006). The Shh and Ihh subgroups are more closely related to each other than to the Dhh subgroup that is closest to Drosophila $\mathrm{Hh}$ (Varjosalo and Taipale, 2008).

The growth of a skeletal element depends on the precise regulation of chondrocyte proliferation and hypertrophy. Appropriate morphogenesis requires integration of proliferation and hypertrophy over the entire element to form the long axis along which the tissue growth follows (Karp et al., 2000). As a member of the conserved hedgehog family of signaling molecules (Echelard et al., 1993), I hh regulates several aspects of bone formation and development (Amizuka et al., 1996; Seki and Hata, 2004; Vortkamp et al., 1996), and play a critical role for skeleton morphogenesis in vertebrates (Karp et al., 2000). For instance, Ihh is initially expressed within the cartilage primordium of the long bones in mice, and becomes localized in a zone of postmitotic and pre-hypertrophic chondrocytes immediately adjacent to the region of proliferating chondrocytes at birth (Bitgood and McMahon, 1995; Lanske et al., 1996; St-J acques et al., 1999; Vortkamp et al., 1996). Ihh knock-out in the embryogenesis of mice can lead to severe skeletal malformation (St-J acques et al., 1999).

Golden Pompano belongs to the family of Carangidae and is a good candidate species for aquaculture due to fast growth and suitability for cage culture (Ma et al., 2014a). In golden pompano Trachinotus ovatus, over $33 \%$ of fish in a population exhibited at least one type of malformation during the larval period ( $\mathrm{Ma}$ et al., 2014b; Zheng et al., 2014). To understand the cause of malformation in this species, it is necessary to identify an indicator to conduct a rapid and reliable evaluation for predict malformation. As a gene relevant to early bone development, the understanding of the expression of Indian Hedgehog gene (Ihh) during fish ontogeny will provide knowledge to rectify fish malformation during early development. This study was designed to explore the expression of I hh during the ontogeny of golden pompano larvae in the first 18 days post-hatch (DPH), and the response of Ihh to water temperature at 12 and $18 \mathrm{DPH}$. The expression pattern of Ihh would provide essential information on the osteogenesis of golden pompano larvae. Such knowledge can improve the understanding on the bone formation of golden pompano, and provide a potential indicator to predict skeleton malformation of fish larvae in early life.

\section{Materials and Methods}

Expression of Ihh gene in the first 18 days of golden pompano larvae

Fertilized eggs of golden pompano were obtained from Guanghui Aquaculture Hatchery, Hainan Provence, P.R. China, and were transported to Lingshui Town and hatched in 500- $\mathrm{L}$ fiberglass incubators at $26.5{ }^{\circ} \mathrm{C}$ with a hatching rate of $97.5 \pm 1.5 \%$ (mean \pm SD). On 2 DPH, larvae were stocked into three 1000-L larval rearing tanks. Larval rearing tanks were supplied with filtered seawater $(5-\mu \mathrm{m}$ pore size) from the bottom of each tank through upwelling with a daily exchange rate of $200 \%$ tank volume. Water was discharged through an outlet screen $(300 \mu \mathrm{m})$ on the upper side of each tank, and the screen was daily cleaned to reduce clogging. Two air stones were used in each tank to maintain dissolved oxygen close to saturation. Light intensity was maintained at 2,400 lux, and the light regime was controlled at $14 \mathrm{~h}$ light and $10 \mathrm{~h}$ dark. The salinity was maintained at $33 \pm 0.8 \%$ and water temperature was $26.5 \pm 1.0{ }^{\circ} \mathrm{C}$ throughout the experiment. 
Molecular cloning of Indian hedgehog gene in golden pompano larvae at different temperatures

3

Rotifers Brachionus rotundiformis at a density of 10-20 ind $\mathrm{ml}^{-1}$ were used to feed the larvae from $2 \mathrm{DPH}$ to $10 \mathrm{DPH}$. Rotifers fed with baker yeast were enriched with DHA protein Selco (INVE Aquaculture, Salt Lake City, UT, USA) for $12 \mathrm{~h}$ before they were added into the larval rearing tanks. Instant microalgal paste (Nannochloropsis sp.) was also added into larval fish tanks to create a green-water background. Artemia nauplii were first introduced at 0.1 nauplii $\mathrm{mL}^{-1}$ on $10 \mathrm{DPH}$, and then added with a daily increment of $90 \%$ by number. After five days co-feeding, Artemia nauplii were gradually phased out at a daily reduction of $20 \%$ by number until the co-feeding period ended. Artemia nauplii were enriched with DHA Protein Selco (INVE Aquaculture, Salt Lake City, UT, USA) following the manufacturer's instruction.

Response of Ihh gene to rearing temperature

Fertilized eggs of the same batch were obtained from Lingshui, Hainan Province, and transported to the Tropical Fisheries Research and Development Center, South China Sea Fisheries Research Institute, Chinese Academy of Fishery Science, Xincun Town. Upon arrival, all eggs were transferred into 500- $\mathrm{L}$ incubators and hatched at $26{ }^{\circ} \mathrm{C}$. The experimental design included three constant temperatures 23,26 , and $29{ }^{\circ} \mathrm{C}$ with three replicates each. On 2 days post hatch (DPH), yolk sac larvae were acclimatized at each desired temperature for $5 \mathrm{~h}$, and then stocked in 500-L fiberglass tanks at a density of 60 fish $\mathrm{L}^{-1}$. Except for the rearing temperature, all the feeding protocols and rearing conditions were the same as in experiment I.

Total RNA extraction and reverse transcription

On $0,1,2,3,4,5,12$, and $18 \mathrm{DPH}$, approximately $300 \mathrm{mg}$ (wet weight) fish larvae were sampled from rearing tanks in triplicates. Approximately 50 individuals were collected in triplicate on $18 \mathrm{DPH}$. A total of 100 individuals were collected in triplicate, and examined under a dissecting microscope for tissue expression analysis. Total RNA was extracted using TRIzol (Invitrogen, USA). RNA integrity was verified by electrophoresis on a formaldehyde-agarose gel (1.2\%). Absorbance measured the RNA concentration at $260 \mathrm{~nm}$ and the purity was determined at the ratio of absorbance at 260 $\mathrm{nm}$ and $280 \mathrm{~nm}(260 / 280)$ and agarose gel electrophoresis. RNA was reverse-transcribed to cDNA with oligo (dT) primers using a PrimeScript 1st strand CDNA synthesis kit (TaKaRa Biotechnology, Dalian Co., Ltd). The CDNA was used as a template in subsequent PCR.

Cloning of the gene CDNA and real-time PCR

Based on unpublished golden pompano transcriptome sequences measured previously in our laboratory (Illumina HiSeq2000, annotated by NR, KOG, kegg, and Swissprot), the genes cloning primers were designed (Table 1) with Primer 5.0 (Premier Biosoft International, Palo Alto, CA, USA). The PCR reaction systems were; $1 \mu \mathrm{L}$ of golden pompano larval CDNA, $1 \mu \mathrm{L}$ of gene-specific forward primer $(F), 1 \mu \mathrm{L}$ of gene-specific reverse primer $(\mathrm{R}), 0.5 \mu \mathrm{L}$ of ExTaq, $5 \mu \mathrm{L}$ of PCR buffer, $4 \mu \mathrm{L}$ of dNTP mixture $(2.5 \mu \mathrm{M})$ and $37.5 \mu \mathrm{L}$ of $\mathrm{ddH}_{2} \mathrm{O}$ were mixed in a total volume of $50 \mu \mathrm{L}$. The PCR conditions were denaturation at $94{ }^{\circ} \mathrm{C}$ for $1 \mathrm{~min}, 35$-cycles of $94{ }^{\circ} \mathrm{C}$ for $30 \mathrm{~s}$, annealing temperature of each gene for $30 \mathrm{~s}, 72{ }^{\circ} \mathrm{C}$ for $4 \mathrm{~min}$, followed by a $10 \mathrm{~min}$ extension at $72{ }^{\circ} \mathrm{C}$. The PCR products were cloned into the PMD-19T vector (TAKARA, Japan), and sequenced.

Table 1 Sequences of primers used in this study

\begin{tabular}{lcc}
\multicolumn{1}{c}{ Primers } & Sequence(5'-3') & Amplicon seizes (bp) \\
\hline Ihh-F & CAGTCACGGACGATGGAG & 1484 \\
Ihh-R & GCTGTGGTCTGTGCTTTAGT & \\
Ihh-qF & TACGAGTCCAAAGCCCACATT & 87 \\
Ihh-qR & AGCATCGCCAGGGAAACA & \\
EF-1a-qF & CCCCTTGGTCGTTTGCC & 101 \\
EF-1a-qR & GCCTTGGTTGTCTTCCGCTA & \\
\hline
\end{tabular}

Quantitative real-time PCR ( expression in golden pompano larvae. Gene-specific primer pairs for the Ihh gene (Table 1) were amplified in LightCycler480 II (Roche, Switzerland). EF-1a was used as the 
internal reference and amplified. The cycling conditions for Ihh genes and EF1a were as follows: 1 min at $95{ }^{\circ} \mathrm{C}$, followed by 40 -cycles $95{ }^{\circ} \mathrm{C}$ for $15 \mathrm{~s}$, and $60{ }^{\circ} \mathrm{C}$ for $1 \mathrm{~min}$. Dissociation curves were employed to ensure that only one single PCR product was amplified in each gene reaction. For each test, three replicates were performed. The relative quantification ( $R Q$ ) was calculated using ${ }_{\triangle} \mathrm{CCT}$ (comparative threshold cycle) method $\left(\triangle C T=C T\right.$ of target gene - CT of EF-1a, $\triangle{ }_{\triangle} C T={ }^{\triangle} C T$ of any sample - ${ }^{\circ} \mathrm{CT}$ of calibrator sample). The efficiencies of the primers $(E)$ were $E_{1 h h}=0.9999$.

Statistical analysis

The data are all expressed as mean \pm SD, and compared with one way ANOVA (PASW Statistics 18.0, Chicago, SPSS Inc.). Tukey's test was used for multiple range comparisons with the level of the significant difference set at $P<0.05$. All data were tested for normality, homogeneity, and independence to satisfy the assumptions of ANOVA.

Sequences and phylogenic analysis

BLAST analyzed the Ihh gene cDNA sequences at the National Center for Biotechnology I nformation (NCBI) (http://blast.ncbi.nlm.nih.gov/Blast.cgi). The complete ORF regions and amino acid sequences were deduced with ORF finder (http://www.ncbi.nlm.nih.gov/gorf/gorf.html ). The molecular weight (Mw) and isoelectronic point $(\mathrm{pl})$ of deduced amino acids were computed by the $\mathrm{pl} / \mathrm{Mw}$ tool of ExPASy (http://web.expasy.org/compute pi/). Protein domains were predicted using SMART (http://smart.embl-heidelberg.de/). Multiple sequence alignments of amino acids were performed by ClustalX 2.1. The phylogenetic tree was constructed by the neighborjoining ( $\mathrm{NJ}$ ) method in MEGA 6.0, and the bootstrap values were replicated 1000 times to derive the confidence value for the analysis (Tamura et al., 2013). Pairwise deduced amino acids sequence identity and similarity matrices of the Hh family sequences from various species were performed using Matgat 2.02 (Campanella et al., 2003). Homology modeling obtained the three-dimensional structures of golden pompano Ihh (http://swissmodel.expasy.org/workspace/index.php).

\section{Results}

Cloning and sequencing of golden pompano I hh gene cDNA

The cDNA sequence length of the golden pompano Ihh gene (GenBank accession: KY039316) was $1,484 \mathrm{bp}$ with an open reading frame (ORF) of 1,314 bp, which encoded 437 amino acids (aa), with a calculated molecular weight (Mw) of $48.175 \mathrm{kDa}$ and a theoretical isoelectric point ( $\mathrm{pl}$ ) of 6.22 (Fig. 1). The bioinformatics analysis of the deduced polypeptide sequence revealed several significant domains or motifs (Fig. 2). The deduced amino acid of the Ihh gene contained a signal peptide (1-25 aa) a conserved HH-signal domain (26-186 aa), a Hint- $\mathrm{N}$ domain (198-331 aa) and Hint- $\mathrm{C}$ domain (335-379 aa, Fig. 2). The molecular modeling of the golden pompano $\mathrm{Ihh}$ is shown in Fig. 3. The golden pompano Ihh sequence shared $87.93 \%$ identity with human Indian hedgehog protein (PDB ID: 3k7g.1.A, Fig. 3). 
Molecular cloning of Indian hedgehog gene in golden pompano larvae at different temperatures

5

1 CAGTACGGACGATGGAGCGGGAACTGAACCGG6GAGATACTGAAGACATCTCTCTCOC 60

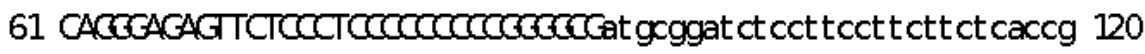

$1 \quad M R$ I S F L L L T A 10

121 cctctctgtgtgccttggtcctcctcctcgcacctgcctcggaggget gcgggccgggga 180

11 S L C A L V L L L A P A S E G C G P G R 30

181 gggggtacggcaagaggcggccccogaagaagct cacgccgctcgcctacaagcagttca 240

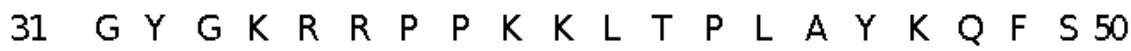

241 gccccaacgttgccgagaagaccctgggagccagcggcagacccgagggcaaaataacgc 300

51 P N V A E K T L G A S G R P E G K I T $R$

301 gcaactccgagcgctttaaagaactgacgocgaattacaacacagacatcatcttcaaag 360

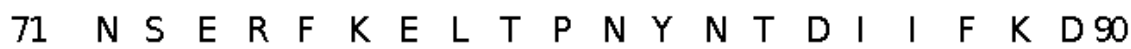

361 at gaggaggacacgggcgccgacaggct cat gacccagcgtt gtaaagacaagttaaact 420

91 E E D T G A D R L M T Q R C K D K L N S 110

421 ctctggccatctctgtgatgaacatgtggccaggtgtgaagct gagagtgacagagggct 480

111 L A I S V M N M W P G V K L R V T E G W130

481 gggat gaggacggccatcattcagaggactcgctgractatgagggacgtgctgtcgaca 540

131 D E D G H H S E D S L H Y E G R A V D I 150

541 tcaccacttcagacagggacagaaataagtatgccatgttggcccggttggct gtagaag 600

151 T T S D R D R N K Y A M L A R L A V E A 170

601 ctggatttgactgggtctactacgagtccaaagcccacattcactgtagcgtcaagtcag 660

171 G F D W V Y Y E S K A H I H C S V K S E 190

661 aacat tctgtggcagccaaaact ggtggttgtttccct ggcgat gctcaggttattctcg 720

191 H S V A A K T G G C F P G D A Q V I L E 210

721 aggacggggctactaaacagatgcgtgaccttcaccct ggtgaccgtgtcttagcttcct 780

211 D G A T K Q M R D L H P G D R V L A S S 230

781 caacagcggatggccacggtcctcttctctacagcccagtct tgtcctttttggacogcc 840

231 T A D G H G P L L Y S P V L S F L D R Q 250

841 agcccaacgtcacaaagatcttctacgtcat tggcaccgacacaggact taatattacgc 900

251 P $N$ N V T T K I F

901 tcacagcagcccacctgatctttgtcacagact gcact ggtggtcagagtgagcct gggt 960

271 T A A H L I F V T D C T G G Q S E P G W290

961 gggaggagactattgaagagccacttttgggctctatact ggggagcaggcogagctggg 1020

291 E E T I E E P L L G S I L G S R P S W E 310

1021 aagcaggtctgaggacagtttttgccagcgaggtccatccaggacagtgtgtacttacgc 1080

311 A $G$ L R T V F A S E V H P G Q C V L T P 330

1081 cgcgagggaaggtggggtcacagacgacattgtcagttgtgacttttgtggaggagcaga 1140

331 R G K V G S Q T T L S V V T $F$ V V E $E$ E $Q R$

1141 ggagcaccggactgtatgcccccctcacccagcatggctctgtagtggtgaacggagtgc 1200

351 S T G L Y A P L T Q H G S V V V N G V L 370

1201 tcgcatcctgctatgct gct gtggacgatcaccatttggcccactgggtcctggccocac 1260

371 A S C Y A A V D D H H L A H W V L A P L 390

1261 tgaggttcttctacagcctgataagaccttcagaactgcagactgacgggctgcactggt 1320

391 R F F Y S L I R P S E L Q T $D$ D G L H W Y 410

1321 atccttggcttctacagaagctagggcaaatgctgetggatgctggacacttccacccct 1380

Fig. 1 Nucleotide sequence and deduced amino acid of Ihh gene from golden pompano Trachinotus ovatus (Linnaeus 1758) 


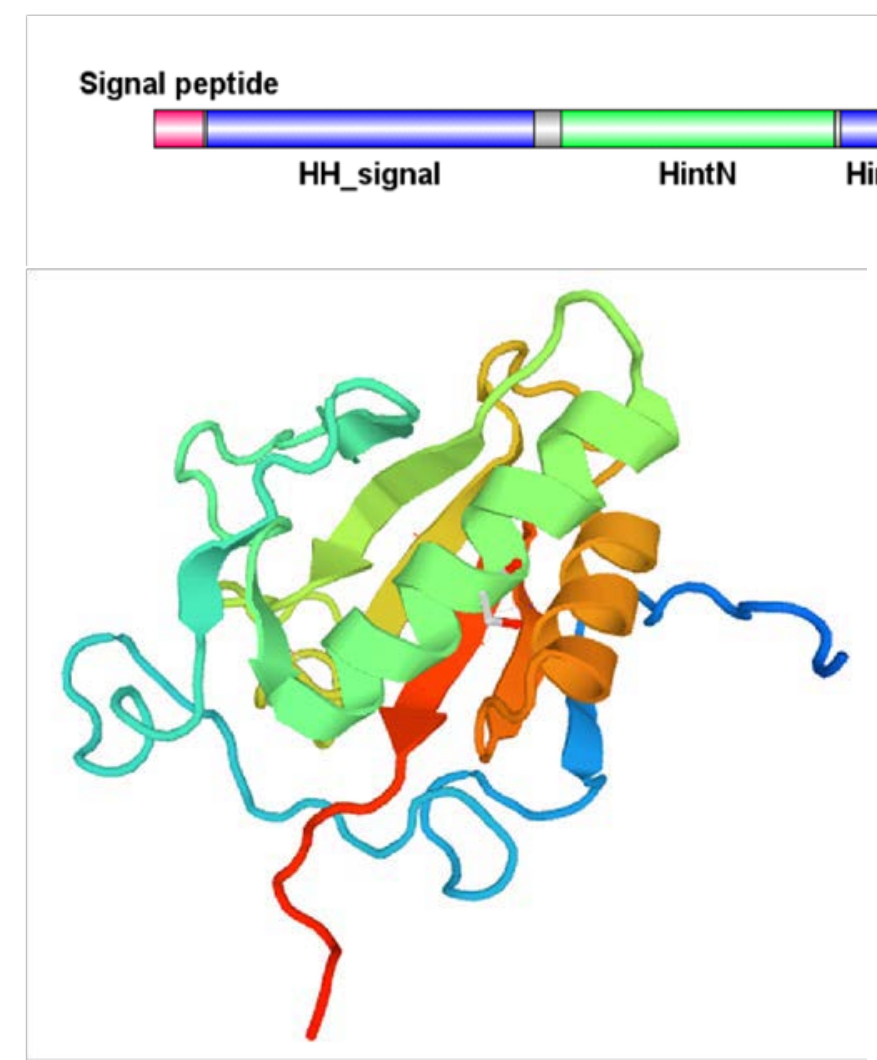

Fig. 3 Predicted tertiary structure of Ihh in golden pompano Trachinotus ovatus (Linnaeus 1758).

Multiple sequence alignments and phylogenetic analysis

Multiple sequence alignment of the deduced amino acid sequences of the Ihh gene with some known Hh family amino acid sequences from various species is shown in Table 2 . The predicted amino acid sequences of the Ihh gene from golden pompano were similar to Lates calcarifer $(93.4 \%$ and 96.1\%, XP_018527527.1) and Takifugu rubripes $(80.9 \%$ and $87 \%$, XP_011609099.1), and different

identity (39.9-65\%) and similarity (58-78.5\%) with other species (Table 2).

Table 2 Identity and similarity between golden pompano I hh with other Hh family homolog.

\begin{tabular}{lllrr}
\hline Genes & Species & GenBank accession & Identity(\%) & Similarity(\%) \\
\hline Ihh & Lates calcarifer & XP_018527527.1 & 93.4 & 96.1 \\
& Takifugu rubripes & XP_011609099.1 & 80.9 & 87 \\
& Larimichthys crocea & KKF30256.1 & 65 & 78.5 \\
& Danio rerio & NP_001030165.2 & 65 & 76.4 \\
& Homo sapiens & NP_002172.2 & 60.8 & 71.4 \\
& Mus musculus & NP_034674.2 & 60.5 & 71.6 \\
& Rattus norvegicus & NP_445836.1 & 60.5 & 71.6 \\
& Xenopus laevis & NP_001079262.1 & 59.4 & 68.2 \\
Dhh & Danio rerio & NP_001025286.1 & 44.1 & 61.7 \\
& Homo sapiens & NP_066382.1 & 49.9 & 64.8 \\
& Rattus norvegicus & NP_445819.1 & 49.7 & 65.2 \\
& Mus musculus & NP_031883.1 & 49.4 & 65 \\
Shh & Amazona aestiva & KQK85399.1 & 58.7 & 72.1 \\
& Gallus gallus & NP_990152.1 & 60 & 71.6 \\
& Monodelphis domestica & NP_001185482.1 & 55.7 & 68.3 \\
& Sus scrofa & NP_001231442.1 & 57.1 & 69.7 \\
& Xenopus laevis & NP_001081782.1 & 54.4 & 70.4 \\
& Danio rerio & NP_571138.1 & 57.2 & 71.2 \\
& Homo sapiens & NP_000184.1 & 57.2 & 69 \\
& Rattus norvegicus & NP_058917.1 & 56.7 & 71.2 \\
& Mus musculus & NP_033196.1 & 57.9 & 71.4 \\
Hedgehog & Drosophila melanogaster & NP_001034065.1 & 39.9 & 58 \\
\hline
\end{tabular}

Expression of Ihh during fish ontogeny 


\section{Molecular cloning of Indian hedgehog gene in golden pompano larvae at different}

temperatures

7

The phylogenetic tree of hedgehogs comprised three main clusters, contain sonic hedgehog (Shh) clusters, Indian hedgehog (Ihh) clusters and desert hedgehog (Dhh) clusters. Hedgehog of Drosophila melanogaster was in the root by outgroup (Fig. 4) and the deduced Ihh amino acid sequences of five fish species contained the signal peptide, and all of them showed high identity and similarity (Fig.5).

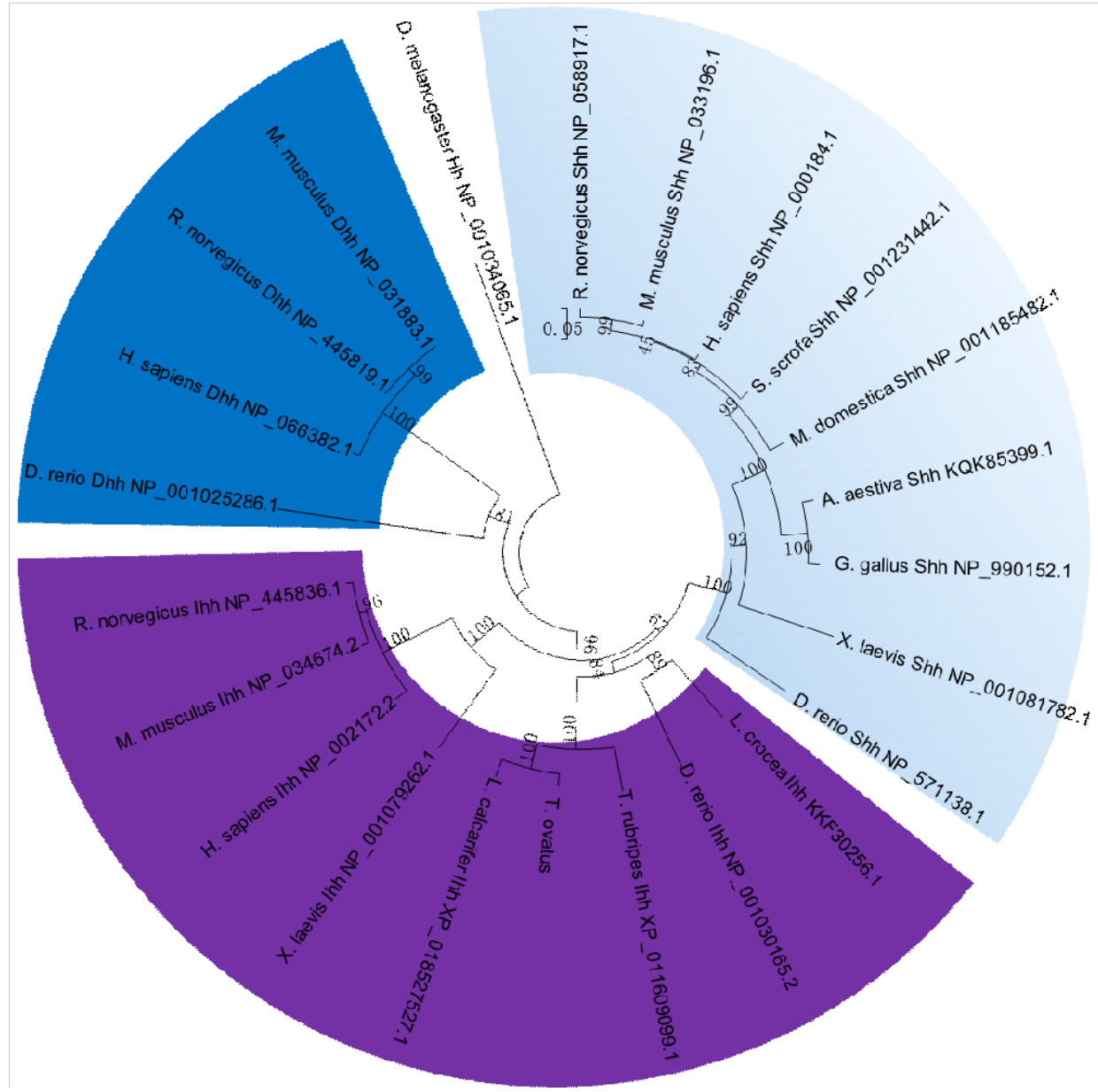

Fig. 4 Phylogenetic tree for amino acid sequences of hedgehog family members.

At hatching, the expression of Ihh in fish larvae was low (Fig. 6). The expression level of Ihh increased with fish age and reached the first peak level on 3 DPH (Fig. 6). Starting from $4 \mathrm{DPH}$, the expression levels of Ihh in fish larvae fluctuated and remained at a relatively high level. On 18 DPH, the expression of Ihh reached the second peak level.

Expression of Ihh in fish tissues

On $18 \mathrm{DPH}$, the highest expression of Ihh in golden pompano was observed in the intestine, followed by the stomach $(P<0.05$, Fig. 7). The expression of $\mathrm{Ihh}$ in the liver of golden pompano was significantly lower than the expression in stomach and intestine $(P<0.05)$. The low expression levels of Ihh in golden pompano were observed in the brain, gills, head-kidney, spleen, and heart on 18 $\mathrm{DPH}$.

Response of I hh to water temperature

Water temperature significantly affected the expression of Ihh in golden pompano on both 12 and $18 \mathrm{DPH}\left(\mathrm{P}<0.05\right.$, Fig. 8). On $12 \mathrm{DPH}$, the expression of Ihh in fish at $23{ }^{\circ} \mathrm{C}$ was significantly higher than those reared in 26 and $29{ }^{\circ} \mathrm{C}(\mathrm{P}<0.05)$. On $18 \mathrm{DPH}$, the expression of $\mathrm{Ihh}$ in fish at $23{ }^{\circ} \mathrm{C}$ was significantly lower than fish at 26 and $29{ }^{\circ} \mathrm{C}(\mathrm{P}<$ 0.05). From 12 to $18 \mathrm{DPH}$, the expression of $\mathrm{Ihh}$ in fish at 26 and $29{ }^{\circ} \mathrm{C}$ increased significantly, while the expression of $\mathrm{Ihh}$ in fish at $23{ }^{\circ} \mathrm{C}$ decreased significantly $(\mathrm{P}<$ $0.05)$ 
T. ovatus

L. calcarifer I th XP_018527527 1

T. rubripes I hh XP 011609099. 11

L. crocea I hh KKF 30256.1

D rerio I hh NP 001030165.2

T. ovatus

L. calcarifer I th XP 018527527

T. rubripes I hh XP 011609099. 1

L. crocea I hh KKF 30256.1

D rerio I hh NP 001030165.2

T. ovatus

L. cal carifer I th XP 018527527

T. rubripes I hh XP 011609099. 1

L. crocea I hh KKF30256. 1

D rerio I hh NP 001030165.2

T. ovatus

L. cal carifer I th XP_018527527

T. rubripes I hh XP 011609099. 1

L. crocea I hh KKF 30256.1

D rerio I hh N_ 001030165.2

T. ovatus

L. calcarifer I th XP 018527527

T. rubripes I hh XP 011609099. 1

L. crosea I hh KKF30256. 1

D rerio I hh NP 001030165.2

T. ovatus

L. calcarifer I th XP 018527527

T. rubripes I hh XP 011609099. 1

L. crocea I hh KKF30256. 1

D rerio I hh NP 001030165.2

T. ovatus

L. calcarifer I th XP 018527527

T. rubripes I hh XP 011609099. I

L. crocea I hh KKF $3 \bar{O}_{256} 1$

D. rerio I hh NP 001030165.2

T. ovatus

L. calcarifer I th XP 018527527

T. rubripes I hh XP 011600099. 1

L. crocea I hh KFF 30256.1

D rerio I hh N_ 001030165.2

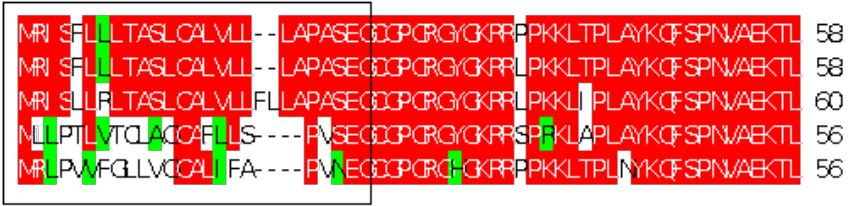

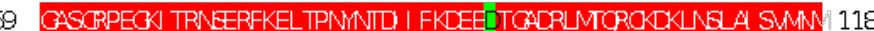
GSC.RPEOK TRNEERFKELTPNMIL I FKDEEDTCACRLMCROLKLELA SMMM 118

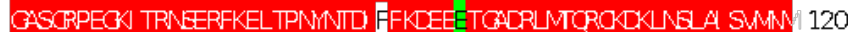

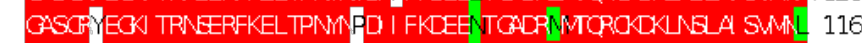

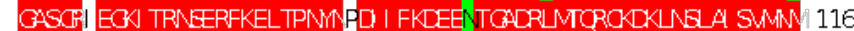

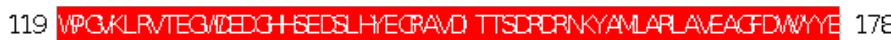

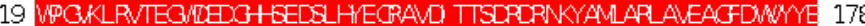

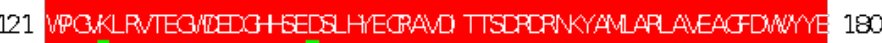

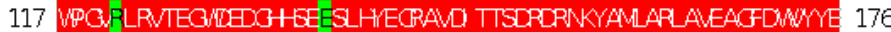

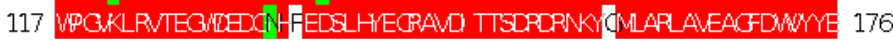
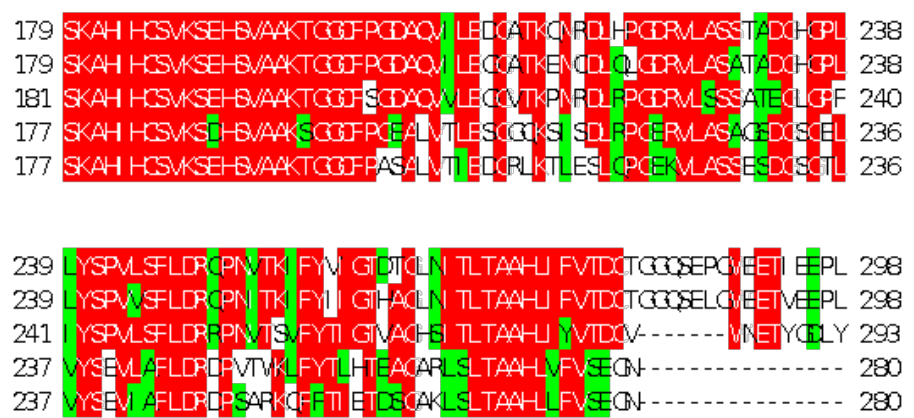

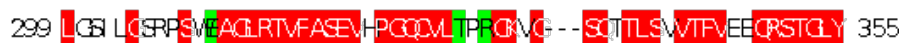

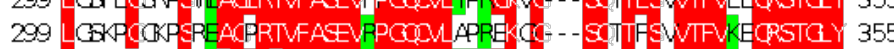

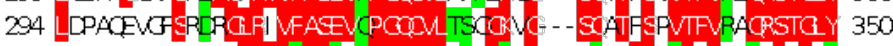
280 - - CSECAMARGLLRTVASDAPPCOU VSECEACOR-LEGLSA TRVT PLEFCAF 337

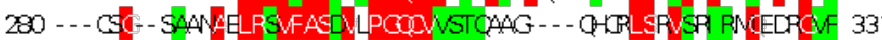

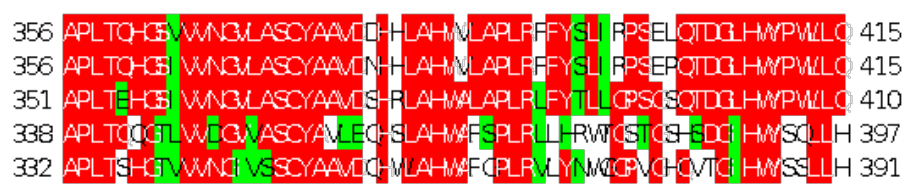

416 KLCMLDAGFPWH EQHF 437

416 KLCOMLAGFHPWH EOHF 437

411 RLCTMLTIEFFPUNDRCQS 432

398 VLCPMLISCPFHPLOMOLR 419

392 WGTQLPAFPPISMIND 413

Fig. 5 Multiple sequence alignment of the deduced amino acid sequence of Ihh with other known homologous Ihh amino acid sequence. 
Molecular cloning of Indian hedgehog gene in golden pompano larvae at different temperatures

9
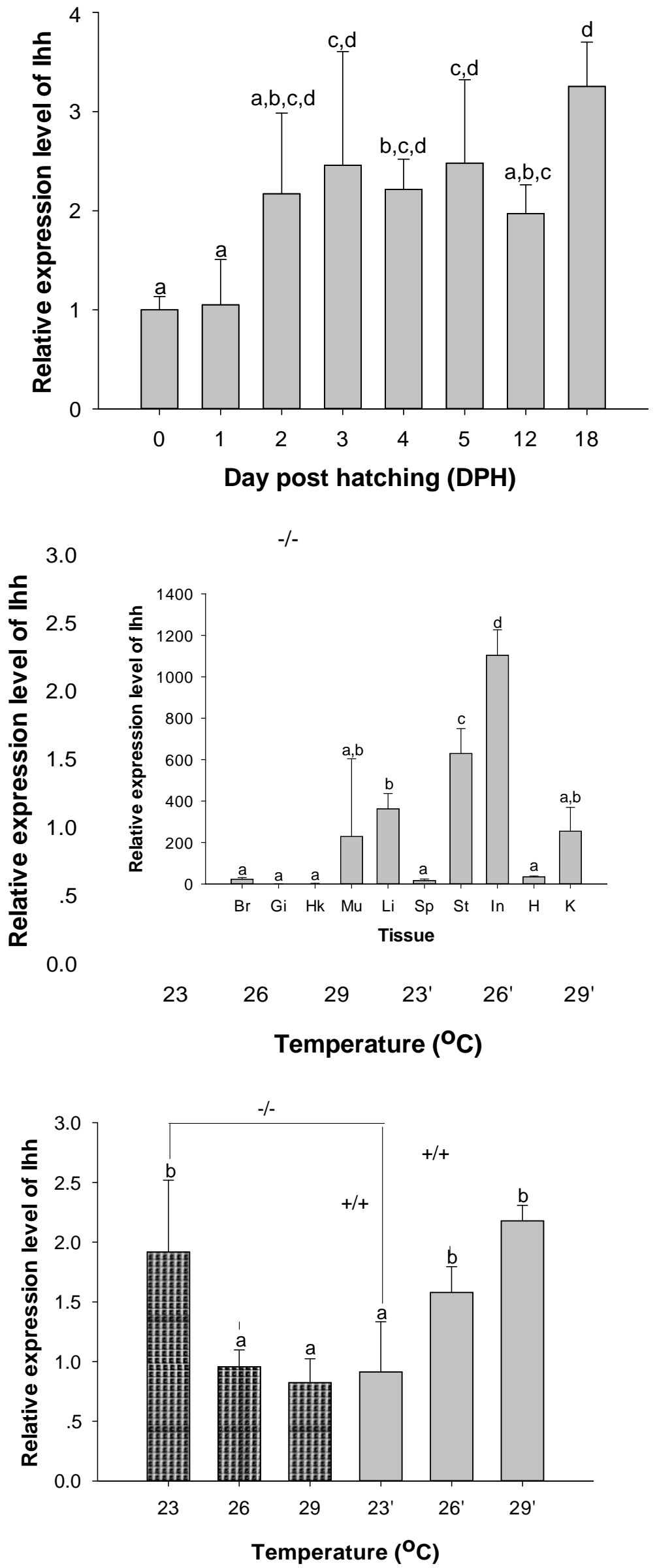

Fig. 6 Relative expression levels of Ihh gene during ontogenetic development of golden pompano larvae. Data with different letters were significantly different $(P<0.05)$

Fig. 7 Relative level of Ihh gene mRNA in different tissues of golden pompano Trachinotus ovatus larvae on $18 \mathrm{DPH}$. Data with different letters were significantly different $(P<0.05)$. Abbreviations: $\mathrm{Br}$, Brain; $\mathrm{Gi}$, Gill; Hk, Head-kidney; Mu, Muscle; Li, Liver; Sp, Spleen; St, Stomach; In, Intestine; $H$, Heart; K, Kidney.
Fig. 8 Relative expression levels of Ihh gene of golden pompano Trachinotus ovatus larvae development at different temperatures on 12DPH $(\because)$ ) and 18DPH ( ). Data with different letters were significantly different ( $P$ $<0.05$ ) 


\section{Discussion}

In this study, the Ihh gene was isolated and identified for the first time in golden pompano larvae. The deduced amino acid of Ihh gene in golden pompano contained a signal peptide (1-25 aa), a conserved HH-signal domain (26-186 aa), a Hint- N domain (198-331 aa) and Hint- $C$ domain (335-379 aa). In bony fish, the sequence of the Ihh gene from golden pompano showed $93.4 \%$ similarity with Lates calcarifer. Similar to other species. Such unique structure in Ihh allows it to produce a mature signaling peptide that can be combined into lipoprotein particles or diffused freely to target cells (Eugster et al., 2007; Ingham and McMahon, 2001). These play a critical role in cell proliferation and cell differentiation in various development processes (Bitgood and McMahon, 1995; Ramalho-Santos et al., 2000; Varjosalo and Taipale, 2008).

Expression of Ihh during fish ontogeny

Previous studies have demonstrated that Ihh gene can participate in the regulation of the development and differentiation of many tissues (Bitgood and McMahon, 1995; Hebrok et al., 2000; I wasaki et al., 1997; Kronmiller and Nguyen, 1996; Ramalho-Santos et al., 2000; Thomas et al., 2000; Vortkamp et al., 1996). Interruption of Ihh can lead to malformation of tissue and organs. For example, increased hedgehog signaling in the pancreas might disrupt organogenesis (Hebrok et al., 2000). Furthermore, loss of hedgehog signaling results in ectopic pancreas formation in chicken embryos (Cooper et al., 1998; Kim and Melton, 1998; Roberts et al., 1995). During bone formation, disruption of Ihh signaling regulation leads to multiple bone diseases, such as progressive osseous heteroplasia (Yang et al., 2015). The expression pattern of Ihh gene during embryonic development has drawn particular attention in the past studies. Bitgood and McMahon (1995) quantified Ihh expression in developing mouse embryos from gestational days 11.5 to 16.5. I hh transcripts present from gestational day 9 to 14 in the mouse mandible (Kronmiller and Nguyen, 1996). In the present study, the expression level of Ihh increased sharply from hatching to $2 \mathrm{DPH}$, and maintained at a relatively high expression level until reaching the peak level on $18 \mathrm{DPH}$. The high level of Ihh expression during early development of larval golden pompano is consistent with the fast growth of this species (Ma et al., 2014a), indicating a fast formation of organs and tissues during this period.

Tissue expression of Ihh in golden pompano larvae

I hh is specifically expressed in some tissues (Varjosalo and Taipale, 2008), such as primitive endoderm (Dyer et al., 2001), pancreas (Kayed et al., 2003), gut (van den Brink, 2007), and prehypertrophic chondrocytes in the growth plate of bones (St-J acques et al., 1999; Vortkamp et al., 1996). Mutation of Ihh in mice can lead to $50 \%$ mortality rate during early embryogenesis, and surviving embryos exhibit cortical bone defects and aberrant chondrocyte development in the long bones (Colnot et al., 2005; St-Jacques et al., 1999). In humans, mutations of Ihh can cause Ihh knock-out pitofemoral dysplasia, a congenital condition characterized by bone defects and short stature (Hellemans et al., 2003). The tissue expression of I hh is species dependent and varies among development times and locations. For instance, $\mathrm{Ihh}$ is expressed mainly in early hypertrophic chondrocytes in the human growth plate (Kindblom et al., 2002). While during the embryonic development of mouse, Ihh shows two main sites of expression: gut endoderm and cartilage (Bitgood and McMahon, 1995). Up to date information on the tissue expression pattern of Ihh in fish is rare. The present study was the first time reporting the tissue expression of I hh in golden pompano larvae. The highest expression of Ihh in golden pompano larvae was observed in the intestine, and followed by stomach on $18 \mathrm{DPH}$. Higher expression in intestine and stomach of fish was similar to the results observed in mouse (Bitgood and McMahon, 1995).

Response of Ihh to water temperature

The critical function of $\mathrm{Hh}$ signaling in bone formation was identified. In the early stages of embryonic limb development, Shh acts as a major morphogen in patterning the limb buds, while Ihh has an essential function in endochondral ossification and induces osteoblast differentiation in the perichondrium (Yang et al., 2015). As primary signaling molecules, Ihh along with hormone-related peptide (PTHrP) plays important roles during 
Molecular cloning of Indian hedgehog gene in golden pompano larvae at different temperatures

11

cell differentiation and skeletal tissue ontogeny (Hogan, 1996; Karp et al., 2000; Yamaguchi et al., 2000). Ihh produced by prehypertrophic and hypertrophic chondrocytes stimulates the production of PTHrP through perichondrial and early chondrocyte cells. PTHrP then maintains chondrocytes in a proliferative and less differentiated state. Once unusually regulation occurs in Ihh, the feedback loop that determines the pace of differentiation of chondrocytes during endochondral ossification is interrupted, and may inhibit osteoblasts from propagation and differentiation to form cartilaginous craniofacial parts of the skeleton (Haga et al., 2003). As a repressor of terminal hypertrophic differentiation (Colnot et al., 2005), I hh was found to be highly upregulated when skeleton malformation occurs in hyperthermic Atlantic salmon (Ytteborg et al., 2010). In the present study, the expression of Ihh in fish reared at 26 and $29{ }^{\circ} \mathrm{C}$ significantly increased as compared to those reared at $23{ }^{\circ} \mathrm{C}$. Such up-regulated expression of Ihh was consistent with the increasing of malformation of golden pompano larvae reared at 26 and $29{ }^{\circ} \mathrm{C}$ (Yang et al., 2016). This may suggest that the expression of Ihh during fish ontogeny could be used as an indicator for skeleton malformation.

In summary, the Ihh cDNA of golden pompano larvae was cloned and analyzed in this study. The present study indicates that the expression of Ihh in golden pompano larvae was significantly affected by the water temperature. The time-dependent expression of Ihh gene in fish larvae is important to understand the ontogenetic development and growth of fish larvae in early life. The monitoring of Ihh gene expressions in golden pompano larvae may serve as a useful indicator in the field and a fish farming setting, leading to a rapid assessment of environmental conditions that affecting fish skeletal development.

\section{Acknowledgements}

This project was supported by National Natural Science Foundation of China (31502186), Pearl River S\&T Nova Program of Guangzhou (201610010166), and Ministry of Human Resources and Social Security of China (2016 High-level Overseas Researcher Come Back to Work Funds).

\section{References}

Amizuka, N., Henderson, J.E., Hoshi, K., Warshawsky, H., Ozawa, H., Goltzman, D. and Karaplis, A.C. 1996. Programmed cell death of chondrocytes and aberrant chondrogenesis in mice homozygous for Parathyroid Hormone-related Peptide gene deletion. Endocrinology 137, 5055-5067.

Beachy, P.A., Cooper, M.K., Young, K.E., von Kessler, D.P., Park, W.J ., Hall, T.M., Leahy, D.J. and Porter, J.A. 1997. Multiple roles of cholesterol in hedgehog protein biogenesis and signaling. Cold Spring Harbor Symposia on Quantitative Biology 62, 191204.

BijIsma, M.F., Speck, C.A. and Peppelenbosch, M.P. 2004. Hedgehog: an unusual signal transducer. BioEssays 26, 387-394.

Bitgood, M.J . and McMahon, A.P. 1995. Hedgehog and bmp genes are coexpressed at many diverse sites of cell-cell interaction in the mouse embryo. Development Biology 172, 126-138.

Burglin, T.R. 2008. The Hedgehog protein family. Genome Biology 9, 241.

Campanella, J.J., Bitincka, L. and Smalley, J. 2003. MatGAT: an application that generates similarity/identity matrices using protein or DNA sequences. BMC Bioinformatics 4, 29.

Chen, M.H., Li, Y.J., Kawakami, T., Xu, S.M. and Chuang, P.T. 2004. Palmitoylation is required for the production of a soluble multimeric Hedgehog protein complex and long-range signaling in vertebrates. Genes Development 18, 641-695.

Chen, X., Tukachinsky, H., Huang, C.H., Jao, C., Chu, Y.R., Tang, H.Y., Mueller, B., Schulman, S., Rapoport, T.A. and Salic, A. 2011. Processing and turnover of the Hedgehog protein in the endoplasmic reticulum. Journal of Cell Biology 192, 825-838.

Colnot, C., de la Fuente, L., Huang, S., Hu, D., Lu, C.Y., St-J acques, B. and Helms, J.A. 2005. Indian hedgehog synchronizes skeletal angiogenesis and perichondrial maturation with cartilage development. Development 132, 1057-1067. 
Cooper, M.K., Porter, J.A., Young, K.E. and Beachy, P.A. 1998. Teratogen-mediated inhibition of target tissue response to Shh signaling. Science 280, 1603-1607.

Dyer, M.A., Farrington, S.M., Mohn, D., Munday, J.R. and Baron, M.H. 2001. Indian hedgehog activates hematopoiesis and vasculogenesis and can respecify prospective neurectodermal cell fate in the mouse embryo Development 128, 1717-1730.

Echelard, Y., Epstein, D.J., St-J acques, B., Shen, L., Mohler, J., McMahon, J.A. and McMahon, A.P. 1993. Sonic hedgehog, a member of a family of putative signaling molecules, is implicated in the regulation of CNS polarity. Cell 75, 1417-1430.

Eugster, C., Panakova, D., Mahmoud, A. and Eaton, S. 2007. Lipoprotein-heparan sulfate interactions in the Hh pathway. Development Cell 13, 57-71.

Haga, Y., Suzuki, T., Kagechika, H. and Takeuchi, T. 2003. A retinoic acid receptorselective agonist causes jaw deformity in the Japanese flounder, Paralichthys olivaceus. Aquaculture 221, 381-392.

Hebrok, M., Kim, S.K., St Jacques, B., McMahon, A.P. and Melton, D.A. 2000. Regulation of pancreas development by hedgehog signaling. Development 127, 49054913.

Hellemans, J., Coucke, P.J., Giedion, A., De Paepe, A., Kramer, P., Beemer, F. and Mortier, G.R. 2003. Homozygous mutations in IHH cause acrocapitofemoral dysplasia, an autosomal recessive disorder with cone-shaped epiphyses in hands and hips. The American J ournal of Human Genetics 72, 1040-1046.

Hogan, B.L.M. 1996. Bone morphogenetic proteins: multifunctional regulators of vertebrate development. Genes Development 10, 1580-1594.

I ngham, P.W. and McMahon, A.P. 2001. Hedgehog signaling in animal development: paradigms and principles. Genes Development 15, 3059-3087.

I wasaki, M., Le, A.X. and Helms, J.A. 1997. Expression of Indian hedgehog, bone morphogenetic protein 6 and gli during skeletal morphogenesis. Mechanisms of Development 69, 197.

Karp, S.J., Schipani, E., St-J acques, B., Hunzelman, J., Kronenberg, H. and McMahon, A.P. 2000. Indian Hedgehog coordinates endochondral bone growth and morphogenesis via Parathyroid Hormone related-Protein-dependent and -independent pathways. Development 127, 543-548.

Kayed, H., Kleeff, J., Keleg, S., Buchler, M.W. and Friess, H. 2003. Distribution of Indian Hedgehog and its receptors patched and smoothened in human chronic pancreatitis. Journal of Endocrinology 178, 467-478.

Kim, S.K. and Melton, D.A. 1998. Pancreas development is promoted by cyclopamine, a hedgehog signaling inhibitor. PNAS 95, 13036-13041.

Kindblom, J.M., Nilsson, O., Hurme, T., OhIsson, C. and Sävendahl, L. 2002. Expression and localization of Indian hedgehog (Ihh) and parathyroid hormone related protein (PTHrP) in the Human growth plate during pubertal development. Journal of Endocrinology 174, R1-R6.

Kronmiller, J.E. and Nguyen, T. 1996. Spatial and temporal distribution of Indian Hedgehog mRNA in the embryonic mouse mandible. Archives of Oral Biology 41, 577583.

Lanske, B., Karaplis, A.C., Lee, K., Luz, A., Vortkamp, A., Pirro, A., Karperien, M., Defize, L.H.K., Ho, C., Mulligan, R.C., Abou-Samra, A.B., J upper, H., Segre, G.V. and Kronenberg, H.M. 1996. PTH/PTHrP receptor in early development and Indian hedgehog-regulated bone growth. Science 273, 663-666.

Lee, J J ., Ekker, S.C., von Kessler, D.P., Porter, J.A., Sun, B.I . and Beachy, P.A. 1994. Autoproteolysis in hedgehog protein biogensis. Science 266, 1528-1537.

Ma, Z., Guo, H., Zheng, P., Wang, L., Jiang, S., Qin, J.G. and Zhang, D. 2014a. Ontogenetic development of digestive functionality in golden pompano Trachinotus ovatus (Linnaeus 1758). Fish Physiology and Biochemistry 40, 1157-1167.

Ma, Z., Zheng, P., Guo, H., Zhang, N., Jiang, S., Zhang, D. and Qin, J.G. 2014b. Jaw malformation of hatchery reared golden pompano Trachinotus ovatus (Linnaeus 1758) larvae. Aquaculture Research 47, 1141-1149. 
Molecular cloning of Indian hedgehog gene in golden pompano larvae at different temperatures

13

Ramalho-Santos, M., Melton, D.A. and McMahon, A.P. 2000. Hedgehog signals regulate multiple aspects of gastrointestinal development. Development 127, 2763-2772. Roberts, D.J., Johnson, R.L., Burke, A.C., Nelson, C.E., Morgan, B.A. and Tabin, C. 1995. Sonic hedgehog is an endodermal signal inducing Bmp-4 and Hox genes during induction and regionalization of the chick hindgut. Development 121, 3163-3174.

Seki, K. and Hata, A. 2004. Indian Hedgehog gene is a target of the bone morphogenetic protein signaling pathway. The Journal of Biological Chemistry 279, 18544-18549.

St-Jacques, B., Hammerschmidt, M. and McMahon, A.P. 1999. Indian hedgehog signaling regulates proliferation and differentiation of chondrocytes and is essential for bone formation. Genes Development 13, 2072-2086.

Tamura, K., Stecher, G., Peterson, D., Filipski, A. and Kumar, S. 2013. MEGA6: Molecular Evolutionary Genetics Analysis version 6.0. Molecular Biology and Evolution 30, 2725-2729.

Thomas, M.K., Rastalsky, N., Lee, J.H. and Habener, J.F. 2000. Hedgehog signaling regulation of insulin production by pancreatic beta-cells. Diabetes 49, 2039-2047.

van den Brink, G.R. 2007. Hedgehog signaling in development and homeostasis of the gastrointestinal tract. Physiological Reviews 87, 1343-1375.

Varjosalo, M. and Taipale, J. 2008. Hedgehog: functions and mechanisms. Genes Development 22, 2454-2472.

Vortkamp, A., Lee, K., Lanske, B., Segre, G.V., Kronenberg, H.M. and Tabin, C.J . 1996. Regulation of rate of cartilage differentiation by Indian hedgehog and PTH-related protein. Science 273, 613-622.

Wada, H. and Makabe, K. 2006. Genome duplications of early vertebrates as a possible chronicle of the evolutionary history of the neural crest. International J ournal of Biological Sciences 2, 133-141.

Yamaguchi, A., Komori, T. and Suda, T. 2000. Regulation of osteoblast differentiation mediated by bone morphogenetic proteins, hedgehogs, and cbfal. Endocrine Reviews 21, 393-411.

Yang, J., Andre, P., Ye, L. and Yang, Y. 2015. The Hedgehog signalling pathway in bone formation. International J ournal of Oral Science 7, 73-79.

Yang, Q., Ma, Z., Zheng, P., Jiang, S., Qin, J.G. and Zhang, Q. 2016. Effect of temperature on growth, survival and occurrence of skeletal deformity in the golden Indian J ournal of Fisheries 63, 74-82.

Ytteborg, E., Baeverfjord, G., Torgersen, J., Hjelde, K. and Takle, H. 2010. Molecular pathology of vertebral deformities in hyperthermic Atlantic salmone (Salmo salar). BMC Physiology 10, 1-16.

Zheng, P., Ma, Z., Guo H., Zhang, D., Fu M., Zhang, N. and Jiang, S. 2014. Osteological ontogeny and malformations in larval and juvenile golden pompano Trachinotus ovatus (Linnaeu 1758). Aquaculture Research 47, 1421-1431. 